\title{
FLEXIBLE SCHEDULING TRAFFIC FOR VIDEO TRANSMISSION IN PROFIBUS NETWORKS
}

\author{
Silvestre, J. * Sempere, V. ${ }^{* *}$ \\ *Dpto.Computer Engineering.UPV.jsilves@disca.upv.es \\ ** Dpto. Communications.UPV. vsempere@dcom.upv.es
}

\begin{abstract}
The mechanisms for static scheduling are inefficient and inadequate when the traffic being carried varies greatly in its bandwidth requirements, especially if there are also variations in run time. For this reason, dynamic traffic scheduling over Profibus, with the objective of developing flexible scheduling systems over this network is analyzed. This will allow us to address a scenario where the traffic load can vary over time. As a typical example of this scenario, the transmission of video sequences for monitoring applications has been selected, where the bandwidth presents important time variations. With the application of the proposed scheme, we will obtain flexibility in the network traffic scheduler, and also, the throughput of the video traffic is improved with respect to static scheduling. Copyright (c)2003 IFAC
\end{abstract}

Keywords: Networks, Scheduling, Monitoring elements, Image flows

\section{INTRODUCTION AND PREVIOUS WORK}

The traffic scheduling schemes for DCCS (Distributed Computer Control Systems) applications where real-time is an important characteristic, usually employ static schemes which demand a prior knowledge of all the characteristics of the process. Although this is adequate and/or usual for hard-real time systems this is not so when speaking of soft real-time processes or when the complexity of the interactions between different nodes cannot be known in advance, or when traffic flows with large variations in bandwidth requirements are in the network. Therefore flexible scheduling systems are needed. This is becoming more and more important in industrial systems (Stankovic, 1996) (Thomesse, 1998) which need mechanisms that permit the behavior of the traffic scheduler to adapt to the traffic requirements

\footnotetext{
1 supported by UPV (Polytechnic University of Valencia. Spain)
}

continuously. This demands the implementation of dynamic scheduling systems which allow the adjustment of the resources used at each moment by each of the elements implicated in a DCCS system, satisfying at all times the time restrictions of the real-time traffic.

Almeida (Almeida, 2001; Almeida et al., 2002b) analyzes in detail this need, proposing several examples, also addressing the dichotomy existing in the current fieldbuses between the flexibility/timeliness behavior and the capacity to satisfy the synchronous/asynchronous traffic requirements. Another interesting aspect proposed by Almeida, although not analyzed in this paper is the definition of QoS parameters in control requirements. This allows flexibility of assignment of resources for control traffic in determined situations which thus gives space for other flows of information.

An example of this type of application with flexibility requirements and changes in run time re- 
quirements is the video transmission for monitoring applications. Here the use of video codecs generates frame sequences with different sizes (relation between sizes of $\mathrm{I}$ frames and $\mathrm{P}$ and $\mathrm{B}$ frames), presenting different bandwidth requirements in each moment, which makes its transport complicated in timed token protocols (KeeYin, 1999), which is the case of Profibus.

The system proposed in this paper is also well suited to address the dynamic scheduling of image transport for control applications different to the static scheme presented by the authors in (Sempere and Silvestre, 2001; Silvestre et al., 2002), in spite of the fact that the traffic load requirement for a determined application has an high stability in its traffic requirements. This greatly facilitates the installation and configuration. Also the proposed system is well suited from the point of view of QoS traffic control adjustment.

The flexible scheduling scheme is based on the carrying of synchronous communication in flexible form and with guaranteed timeliness. Almeida (Almeida et al., 2002a) presents a WorldFIP scheduling algorithm, with the property of being usable on-line, although the objective of these algorithms is to improve the response time as much for the synchronous traffic as the asynchronous. Also analyzed is the architecture and management aspects over CAN in the system known as FTT-CAN (Almeida et al., 1999) (Almeida et $a l ., 2002 b)$ for applications embedded in this network. Pedreiras (Pedreiras et al., 2002) presents a complete flexible and dynamic architecture for Ethernet, known as FTT Ethernet, with the objective of adequately satisfying, with flexibility, the typical fieldbus traffic requirements over Ethernet networks, avoiding some of the inconveniences of using this network as a fieldbus.

As for video transmission over Timed Token Networks, Feng (Feng et al., 1996) analyzes the end-to-end delays in a local scenario (only uses the local information of a node to allocate its synchronous bandwidth) where the synchronous server manages the assigned bandwidth between the multiple flows of a node. Zheng (Zheng and Shin, 1993) proposed a local scheme for the scheduling of video traffic, taking into account variations in demand and avoiding the restriction of a deadline of the same period as other previous synchronous (Agrawal et al., 1993) bandwidth assignment schemes(SBA) . However, in spite of the global schemes producing an overhead in the network, Han (Han et al., 2001) showed that optimum local SBA schemes over timed token protocols don't exist. Moreover, given the dynamic nature of multimedia traffic, the parameters of this traffic in execution can be different from those used in its design, and so a management system capable of dynamic adjustment to the requirements of this traffic is necessary (Chan et al., 1995). Kee-Yin (Kee-Yin, 1999) proposes, for a timed token control network FDDI, a regulation scheme to distribute the request in the most suitable way in the bus, avoiding the superimposition of type I frames between different video sources.

The objective of this paper is to analyze the use of a flexible scheduling scheme over a Timed Token network such as Profibus for the transport of realtime control traffic and video traffic, analyzing the different aspects of the viability of its use, as such evaluating the advantages that this scheme can give to a video transmission system for monitoring applications.

Before analyzing the model, and since we are working with video, it is necessary to review some basic concepts. The convenience given by the use of MJPEG codecs for image compression when they are going to be used in artificial vision processes has been analyzed by the authors in (Sempere and Silvestre, 2003). However, when the images are going to be used for monitoring, it is possible to take advantage of the greater compression capacity which video codecs offer which make use of temporal redundancy of the images (Wu and Irwin, 1998).

Within these codecs three types of frames usually exist. The I frames or intra-frames are coded as still images, not using a past history, and thus can be decoded without the need for any other frame. The compression ratio for these frames is the lowest of all frame types. The $\mathrm{P}$ frame or predicted frames are predicted from the most recently constructed I or P frame. By exploiting temporal redundancies, these frames achieve higher compression ratios than the I frames. Finally there are B frames, or bidirectional frames. These are predicted from the nearest two I or P frames; one in the past and one in the future. They have the highest compression ratio among all frame types. The order of the frames obtained with the codec follows usually the pattern IBBPPBBPBB... and repeats itself. This sequence is called Group of Pictures (GOP).

\section{MODEL}

The proposed system to obtain a flexible scheduling scheme over Profibus, has the objective of realizing an optimum ${ }^{2}$ (Han et al., 2001) and centralized (see Fig.1) SBA. The stations make their traffic requirement request to the central

\footnotetext{
2 SBA scheme that finds a feasible set of bandwidth allocation subject to the protocol and deadline constraints whenever such a set exists
} 


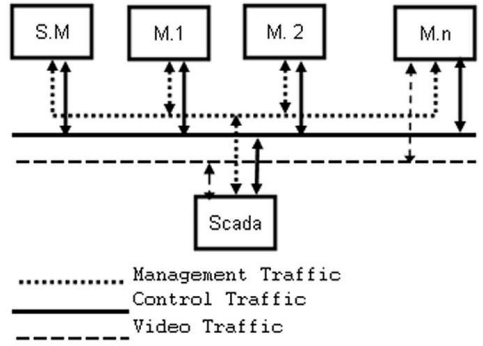

Fig. 1. Architecture of proposed system

station know as Scheduler Master SM (as a LAS in Foundation Fieldbus H1). The SM allocates access rights according to the request received and the requirements of the other network stations $\left(M_{1} t o M_{n}\right)$ existing a station (SCADA) where the video flows are visualized and where the human operator acts according to Chan's model (Chan et al., 1995) being responsible for achieving management objectives. That is, providing network services that meet the need of customer applications and defining resource allocation strategies that provide benefits for the service provider.

\subsection{Cycles considered}

\section{Elementary Cycles}

In this type of scheduling the Elementary Cycle (EC) is seen as a bus time window used to exchange the traffic associated with each EC (see Fig.2a). The duration of the EC is typically set to the highest common factor (HCF) of the periods of synchronous traffic flows. In this type of scheduling the macrocycle (MC) is defined as the period of time in which the synchronous requests are repeated, derived therefore from the cyclical orders and with a period equal to the least common multiple (LCM) of the periods of traffic flow (Fig.2a).

Often many of the proposed scheduling schemes, work in a static way with two windows of fixed size existing for all the EC's, one for the synchronous traffic and another for the asynchronous traffic (HS and HA in Fig.2a). In these cases, within the window size for synchronous traffic, the peak is used in order to guarantee its QoS parameters, and the rest of the EC is used for asynchronous traffic. This scheduling scheme can be very inefficient in its use of the available bandwidth, given that the difference between the maximum peak of synchronous traffic in a EC and the synchronous traffic of each EC in the MC can be significant, in this way introducing a large number of unused slots. At the moment of introducing multimedia traffic, some authors add another window for this, using a fixed scheduling scheme (Tovar et al., 2001) of the Equal Partition Allocation Scheme type. In our opinion the throughput of

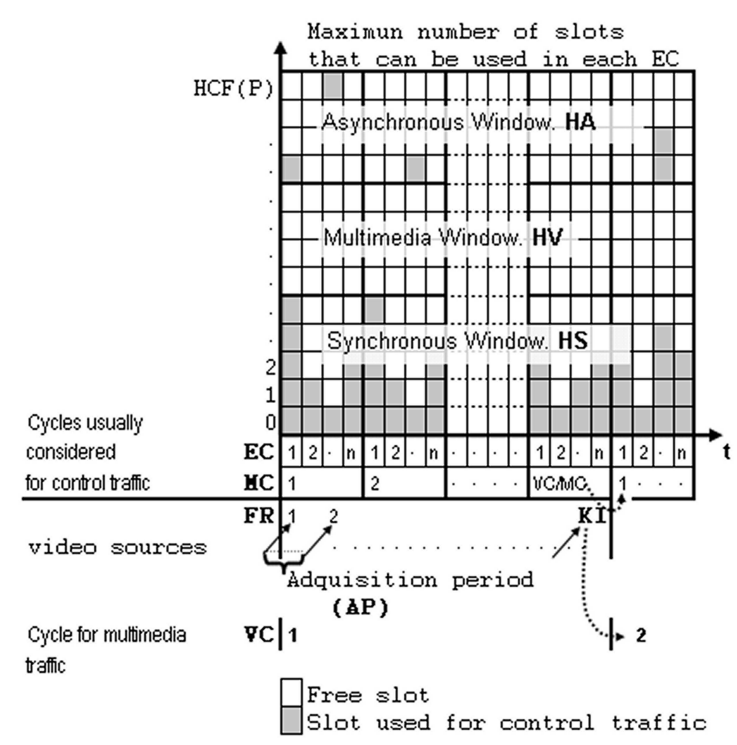

a) Fixed windows

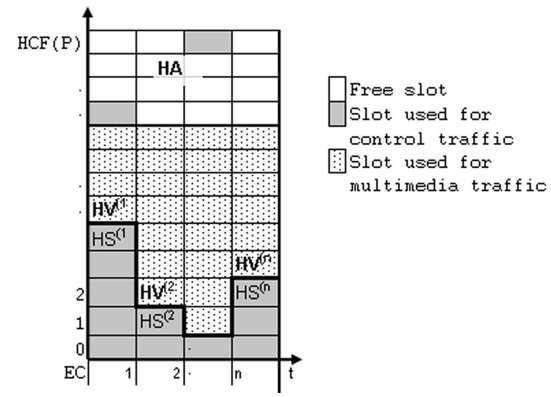

b) Variable and dynamic windows

Fig. 2. Cycles considered in the paper

multimedia transmission can be significantly improved through the use of a scheduling system which takes into account the dynamic characteristics of the video traffic.

In the proposed scheme we provide a mechanism which permits that the window sizes are not fixed but variable in the functions of the EC (Fig.2b). This will permit the optimization of bandwidth use and the capacity to adapt on-line to changes in the traffic sources requirements.

\section{Video Cycles}

To give support to the video traffic scheduling the Video Cycle (VC) is introduced. This presents a period depending on the characteristics of the frame acquisition system of the period of image acquisition (Know as AP, and usually in msec.) and the interval between keyframes (KI, usually 24-30 frames) being:

$$
V C=A P x K I
$$

When this time has elapsed, the IPB frame pattern will keep repeating.

In fig.2a we can see the way in which the period between two frame requests (FR) can encompass several EC's. This will be taken advantage of by 
the scheduling algorithm when placing the load of each frame, given that a AP deadline will be considered for each frame.

\subsection{Traffic Model}

In the proposed system various types of traffic flows have to coexist. The existence of $k$ stations, $p$ synchronous real-time control flows in each station and $m$ video flows is assumed.

\section{Real-Time Control Synchronous Traffic}

Each station $i(i \leq k)$ has $j(j \leq p)$ flows of synchronous messages $S_{i}^{(j}$ characterized by its period $P_{i}^{(j}$ and its deadline $D_{i}^{(j}$. It is assumed that the messages are of the maximum length provided by the protocol. $\left(C_{\max }=240\right.$ bytes in Profibus)

And that the deadline of each $S_{i}^{(j}$ is not $P_{i}^{(j}$ (which can introduce a high variability when considering the highest periods) but a determined number of EC's, in fact 1 EC.

We denominate as $H S_{i}^{(v}$ the space reserved in the station $i$ for the synchronous real-time control traffic in the $E C v$. In order to satisfy the requirements of this traffic it is necessary to assign to each station with traffic control a synchronous capacity sufficient for the transmission of all the synchronous requests of the $E C v$, that is to say:

$$
\begin{array}{r}
H S_{i}^{(v} \geq \sum_{j=1}^{p} e C_{i}^{(j}, \text { where } \\
e=1, \text { ifvMOD }\left(P_{i}^{(j} / H C F(P)\right)=0, \\
e=0, \text { inothercases }
\end{array}
$$

We denominate as $H S^{(v}$ the space reserved in the $E C v$ for the transmission of all the synchronous requests of all stations, that is to say:

$$
H S^{(v}=\sum_{i=1}^{k} H S_{i}^{(v}
$$

\section{Real-Time Control Asynchronous Traffic}

Each station or node $i$ can generate any given asynchronous messages at any instant characterized by a deadline $D_{i}^{\text {(asyn }}$. It is assumed in the paper that all the asynchronous messages have the same deadline $D^{(\text {asyn }}$. Given that this type of traffic presents a low load, such as that typically used in alarms, etc. its duration $C A_{i}$ in all stations is limited to $C A$ bytes. In each EC a space will be reserved for the transmission of an asynchronous message in each station, denominated HA.

$$
H A \geq \sum_{i=1}^{k} C A
$$

\section{Real-Time Video Traffic}

Each one of the video flows $\mathrm{m}$ is characterized not only by the $A P$ and $K I$ previously mentioned but also by a quality index which determines frame load $\left(C I_{(m}, C P_{(m}, C B_{(m}\right)$, as well as its distribution in a MPEG4 sequence (the order of the $\mathrm{p}$ frames type $\mathrm{P}$ and $\mathrm{b}$ frames type $\mathrm{B}$ which follow frame I). It will be necessary in order to transport these flows to associate with each one, the video windows, know as $H V_{(m}$, of different size in the function of the $E C$ and of the frame type, for each of the $E C^{\prime} s$ that there are between two type I frames (that is to say, for $V C$ )

$$
H V_{(m}=\left\{H V_{(m}^{1}, H V_{(m}^{2}, \ldots, H V_{(m}^{V C}\right\}
$$

in a way that each one of the frames can be delivered before its AP. Therefore, so that all the frames of a GOP can be transmitted before their deadline, the following must be fulfilled:

$$
\begin{array}{r}
H V_{(m}^{1}+H V_{(m}^{2}+\ldots+H V_{(m}^{V C} \geq \\
C I_{(m}+C P_{(m}+C B_{(m}
\end{array}
$$

whereas to meet the deadline of each frame, transmitted between AP EC's the following must be fulfilled:

$$
H V_{(m}^{n}+H V_{(m}^{n+1}+\ldots .+H V_{(m}^{n+A P} \geq C_{(m}
$$

being $\mathrm{n}$ an EC multiple of the $\mathrm{AP}$, and $\mathrm{C}$ the load of the frame I, $\mathrm{P}$ or $\mathrm{B}$ in function of the IPB distribution of this sequence and of the EC in which we find ourselves.

The window for the video traffic in each $E C$ will then be the sum of the windows associated with each of the flows being:

$$
H V^{(v}=\sum_{i=1}^{m} H V_{(i}^{v}
$$

\section{Scheduler Traffic}

For the management of the scheduling policy, it is necessary to transfer request/response messages. These messages have a maximum length of $C A$ bytes and the deadline is associated with the type of message it is dealing with. The same as in the case of asynchronous traffic, a $H M$ window (Management Window) could be reserved for the transmission of this type of message. However, in this paper it is considered unlikely that the need exists to transmit at the same instant as much management traffic as asynchronous traffic through the same window used by both types of transmission. Nevertheless for the management of the protocol, it is vital that there is transmission of control packets for the management cycle, in order that the scheduler can guarantee the trans- 
mission of at least one type 5 message (see table 1 ) know as HMC (cycle management) for each EC.

\subsection{Bandwidth limitation}

In a token pass protocol, a parameter that is fundamental and common to all stations is the Token Token Rotation Time (TTRT), which shows the passage of time between two consecutive token arrivals. Its selection influences the time in which each station can keep the token (Agrawal et al., 1993), in the traffic that can be guaranteed on Profibus, and in the worst case, a high priority package (Tovar and Vasques, 1999). Nevertheless, given the necessity of guaranteeing large -sized flows, an outline is chosen which limits the synchronized capacity of each station, so that with a TTRT (Silvestre et al., 2002)

$$
\begin{aligned}
& T T R T \geq \max _{i=1 . . V C}\left\{H S^{i}\right\}+H A+H V_{i, \forall i=1 . . V C}^{(m} \\
&+\max _{i=1 . . V C}\left\{H V_{i}^{(m}\right\}+H M_{C}+\Theta N(9)
\end{aligned}
$$

it guarantees the maintenance of the space which is assigned to each station, avoiding overrun situations. Furthermore, in order to meet deadlines, the following must be fulfilled:

$$
\begin{array}{r}
H S^{i}+H A+H V_{i}+H M_{C} \\
\leq P_{\text {min }}, \forall i=0 . . V C
\end{array}
$$

which also optimizes the useful bandwidth of Profibus (Silvestre et al., 2002) by being used as a formula to calculate the admission control as well as periodical requests, such as video requests.

\section{PROTOCOL}

\subsection{Set-up and run-time}

The protocol gives greater priority to the control packets so that to begin with, in its initialization phase, only these can be dealt with, to determine thus the basic parameters of the network. In the set-up phase of the system, the SM emits a type 0 broadcast packet (see table 1) indicating the initialization state to the rest of the stations and releases the token. As this reaches the other stations, these will make their request to SM using the transmission of type 1 packets. A part of the SP (Setup Phase) having elapsed, the SM has sufficient information available to calculate the EC and $\mathrm{MC}$, and so assigning the bandwidth required to satisfy all these requests (type 3 packets). Once the initialization phase has finished, the SM starts to generate EC cycles (SDN broadcast packet type 5) where each station transmits a number of
Table 1. Codification of management frames

\begin{tabular}{lccllll}
\hline Function & $\mathrm{T}$ & $\mathrm{C}$ & & & & $\mathrm{NB}$ \\
\hline Initialization & $\mathrm{B}$ & 0 & & & & 1 \\
Sync. Req & $\mathrm{U}$ & 1 & $\mathrm{~A}$ & $\mathrm{~N}$ & period & 4 \\
Vid. Req. & $\mathrm{U}$ & 2 & $\mathrm{~A}$ & $\mathrm{~N}$ & $\mathrm{QR}$ & 6 \\
Answer 1 & $\mathrm{U}$ & 3 & $\mathrm{~N}$ & $\mathrm{EC}$ & & 3 \\
Answer 2 & $\mathrm{U}$ & 4 & $\mathrm{~N}$ & $\mathrm{QA}$ & & 6 \\
EC Change & $\mathrm{B}$ & 5 & $\mathrm{EC}$ no. & & 2 & \\
EC Maint. & $\mathrm{B}$ & 6 & no. free & & 2 \\
Control & - & 7 & & & 1 \\
Video & - & 8 & & & 1 \\
\hline T: Type. C: Code. $N B:$ no. of bytes. B: Broadcast. U: \\
Unicast. A: Address. $N:$ no. request. QR: Quality request. \\
QA: Quality assigned.
\end{tabular}

packets not greater than the bandwidth assigned by the scheduler $\left(H S_{(i}\right.$ and/or $H V_{(i}$, depending on the traffic sources of each master). If all the stations have finished transmitting their assigned traffic and the EC has not yet been completed, the scheduler will transmit type 6 packets indicating the free space which can be used by the other stations for the transmission of new synchronized requests or non-real-time traffic, of new requests or of pending video packets.

\subsection{Video request}

Once the initialization has finished, the monitoring station (SCADA) might wish to visualize video flows in the network with a determined quality. To do this, it transmits type 2 packets to SM, indicating the direction of the video source, the number of the associated requests and the quality parameters of the video. The scheduler will have to analyze if it is possible to deal with the request with the quality asked for. If this is not possible, it will be attended to, with an inferior quality. If this not possible, he will act as an admission control, disabling the transmission of this flow. In the cases where the request can be attended to with an inferior quality, the transmission windows of each type of frame will be set to the station which generated the video, using the transmission of the type 4 packet. The scheduling policy for video traffic is therefore FIFO type, since the requests are attended to according to the order of arrival.

It is assumed that the scheduler has information stored about the history of past sessions and/or by configuration on the index relation of requested quality, and bytes required for the I, P and B frame types. The transmission protocol of the images has been explained in (Sempere and Silvestre, 2001). 
At any moment, using HA, the stations can transmit a type 1 message to ask for the transmission of new flows of periodic traffic or to cancel previous requests (indicating a 0 period ). In its turn, SCADA could add, remove or modify the quality of the video stream, using type 2 packets.

Faced with these requests, SM has to reschedule the synchronous bandwidths associated with each master, using the transmission of 3 and 4 packets. Given the greater priority of control traffic over video traffic, the control requests have to be attended to with greater speed of reply than the video traffic. This does not create any drawback, as each modification in the synchronous traffic involves few changes in the assigned bandwidth, while in the case of video requests, important modifications may be involved, and these must be modified in a synchronized form. Furthermore, with control traffic we are on a msec. timescale, whereas the operator who monitors the video information is on a seconds scale (Lazar and Stadlelr, 1993).

\subsection{Data structure to give support to the protocol}

As a consequence of the requests granted by the scheduler, the masters with control traffic only need to know the bandwidth assigned to each EC (Silvestre et al., 2002)in a MC. The masters with video traffic need to know the EC in which they have to transmit frame type I, the IPB distribution of a $\mathrm{VC}$, and the bandwidth required for the transmission of frames type I, P or B.

\section{SCHEDULING ALGORITHM}

Algorithm 1. Synchronous traffic scheduling

01 if ( state $==\mathrm{SP}$ ) CalcBasicParam () ;

02 for $(i=1 ; i \leq$ NumPer;i++)

03 tper $=$ period $[\mathrm{i}] / \mathrm{EC}$;

04 if $\operatorname{CtrlAdPer}(\mathrm{i})$

05 for $(j=$ tper; $j<E C ; j+=$ tper $)$

$06 \quad \mathrm{HS}[\mathrm{j}]++$

Algorithm 2. Video traffic scheduling

01 for $(\mathrm{i}=1 ; \mathrm{i}=\mathrm{NumMul} ; \mathrm{i}++)$

02 if (CtrlAdMul(i))

03 for $(\mathrm{i}=1 ; 1 ; \mathrm{KI} ; \mathrm{i}+=\mathrm{AP} / \mathrm{EC})$

$04 \quad \mathrm{C}=$ CalcType () ;

$05 \quad$ Free $=$ SlotsFreeRF () ;

$06 \quad$ while $(C>0)$

$07 \quad$ FreeEC $=\max -\mathrm{HS}[\mathrm{i}+\mathrm{t}]-\mathrm{HV}[\mathrm{i}+\mathrm{t}]$;

$08 \quad \mathrm{t}=0$;

$09 \quad$ while $(($ FreeEC $>0) \&(\mathrm{C}>0))$

10 FreeEC--; C--;
The algorithm of control traffic scheduling assumes a deadline of $1 \mathrm{EC}$ in control traffic requests so as not to allow jitter in its transmission and therefore the necessary slots are arranged according to the period of each flow. This produces peaks in the bandwidth demand necessary for the transmission of control traffic at every moment (see example Fig. 3), which has to be taken into account in the scheduling of video traffic.

The scheduling algorithm 1 calculates the basic functioning parameters in each request (line 1,algorithm 1), in other words, the $\mathrm{EC}$ and $\mathrm{MC}$, according to the periods of the received requests in the set-up phase.

Afterwards, for each request made, the admission control (line 4,algorithm 1) verifies the completion of (10) in the ECs of an MC, with the characteristic $H V_{i}=0$. In other words, giving priority to control traffic so that the acceptance of new traffic of this type can reduce the quality of, or simply eliminate, a video flow. Up to now, it had not been foreseen that the introduction of new flows of periodic traffic during the run time could alter the basic parameters, such as the duration of EC and MC.

The scheduling algorithm of video traffic (algorithm 2) is based on the synchronization of type$B$ frames on a type-I frame of the first video request. This limits the number of simultaneous video sources with the distribution seen in point 2 , at 5 sources, which is considered sufficient in an environment of video transmission for monitoring in a Profibus network.

For each video flow, first of all the admission is analyzed, which will be determined according to 10, but now considering $H V_{i}$ if the video flow can be transmitted or not, and with what quality. Furthermore, it is considered that the frame $\mathrm{C}$ load (I, P or B) could be shared out between different ECs, specifically between AP/EC.

Subsequently, the type of packet which is to be sent is calculated according to the request number, which determines the load of the ECs which make up a $\mathrm{AP}$ ( $\mathrm{C}$ in line 4, algorithm 2), and the free slots left by the periodic traffic in the ECs which occupy the frame transmission (line 5). Next, the algorithm shares out the $\mathrm{C}$ load of the frame, in as many ECs as are necessary, assigning the $H V_{(m}^{E C}$ to the video flow. This form of distributing the $\mathrm{C}$ load to each frame, tries to locate it in the fewest ECs possible, instead of using a uniform distribution between the ECs that satisfy a RF, so in cases where the real frame load is higher than the average used, these packets could 
be sent in the last EC's of the RF. This would be carried out in this way in the case where the SM sends type 6 packets with sufficient capacity to send the rest.

\section{NUMERICAL EXAMPLE}

Table 2. Synchronous traffic

\begin{tabular}{llllll} 
Master 1 & & Master 2 & \multicolumn{3}{l}{ Master 3 } \\
\hline no. & $\mathrm{P}$ & no. & $\mathrm{P}$ & no. & $\mathrm{P}$ \\
\hline $1-1$ & 10 & $2-1$ & 10 & & \\
$1-2$ & 30 & $2-2$ & 30 & $3-1$ & 30 \\
$1-3$ & 60 & $2-3$ & 60 & $3-2$ & 60 \\
$1-4$ & 90 & $2-4$ & 90 & $3-3$ & 90 \\
& & & & & \\
Master 4 & & Master 5 & & Master 6 & \\
\hline no. & $\mathrm{P}$ & no. & $\mathrm{P}$ & no. & $\mathrm{P}$ \\
\hline $4-1$ & 30 & $5-1$ & 30 & $6-1$ & 30 \\
$4-2$ & 60 & & & & \\
$4-3$ & 90 & $5-2$ & 90 & $6-2$ & 90 \\
\hline
\end{tabular}

Table 3. Video traffic

\begin{tabular}{lllllll}
\hline & \multicolumn{3}{c}{ high } & \multicolumn{3}{c}{ low } \\
Type & $\mathrm{I}$ & $\mathrm{P}$ & $\mathrm{B}$ & $\mathrm{I}$ & $\mathrm{P}$ & $\mathrm{B}$ \\
\hline Sequence 1 & 52 & 20 & 2 & 36 & 10 & 1 \\
Sequences $2,3,4$ & 36 & 10 & 1 & 23 & 5 & 1 \\
\hline Sizes given in no. of full packets. AP=30 & ms. KI=30 \\
frames. GOP's of the sequences obtained with DivX 3.0: \\
IBPPPPBPPPPBPPPPBPPPPBPPPPBPPP
\end{tabular}

Departing from an initial scenario with the periodic and video traffic shown in tables 2 and 3 (the data of the load of each one of the frames of a video sequence has been extracted from the analysis of 4 sequences of 5040 frames, 168 seconds), the fundamental parameters would be:

$\mathrm{EC}=10$ msec. $\mathrm{MC}=180$ msec. $\mathrm{VC}=900$ msec.

Up to 8.1 msec. is reserved for periodic traffic (including the HMC of each start of a cycle), and the rest, 1.9 minus the token rotation latencies, for management and asynchronous traffic (up to 20 $\mathrm{CA}$ packets $=10$ bytes, which supposes a duration of $90 \mu s e c$.), this is considered sufficient to reserve space for the transmission of an asynchronous packet and a request packet for each station as well as for the transmission of reply 3 and 4 packets for part of the SM. (The parameters of the Profibus network as well as the calculation of time can be found in (Silvestre et al., 2002).

This will generate distribution for synchronized traffic as can be seen in Fig. 3 With this distribution, the requirements of this type of traffic are guaranteed, a free space would also exist, that in the case of static assignation outlines, would serve for the transmission of 8 complete packets (slots 19 to 26 in Fig. 3).

Nevertheless, using a static outline (given the maximum peak of periodic traffic of 18 complete packet that are given in the $18 \mathrm{ECs}$ ), it is easy to see how the transmission of frame I of one type1 sequence would require almost 9 ECs (only 8 slots per EC can be used), therefore losing frames 2 and 3. For the transmission of a type-P packet, 3 ECs are needed, and 1 EC for a type B packet. Nevertheless, adding whatever other video flow in these conditions will worsen the situation, causing the sequences to lose a large number of frames as well as being taken to a level of application with important delays, which could produce glitches in the resulting video.

By means of the scheduling outline proposed, four video sequences could be transmitted, using high quality in the first two sequences, and low quality in the last two. This would avoid loss of frames and delays in the deadline of each frame, as can be seen in Fig. 3

\section{CONCLUSION AND FUTURE RESEARCH}

The dynamic scheduling outline proposed allows the maximization of the number and quality of video sequences which can be transmitted, guaranteeing the behavior of real-time traffic whether periodic or not. Although it had a different aim from the monitoring, by the use of a static assignation outline, such as that used by the authors (Sempere and Silvestre, 2001; Silvestre et al., 2002), with the scenario of the numerical example explained, the valuation of transmission of images is $1.53 \mathrm{Mbps}$, whereas in this way 2.15 Mbps is reached by inputting only one overhead of 1 byte in each packet. Nevertheless, the most important characteristic is to have the flexibility to change the configuration as well as the video and control traffic on-line.

\section{REFERENCES}

Agrawal, G., B. Chen and W. Zhao (1993). Guaranting synchronous message deadlines with the timed token medium access control protocol. Proc. of ACM Multimedia.

Almeida, L. (2001). Flexibility, timeliness and efficiency in fieldbus systems: the disco project. Proc. 2001 8th IEEE Int. Conf Emerging Technologies and Factory Automation 1, 159167.

Almeida, L., E. Tovar, J.A. Fonseca and F. Vasques $(2002 a)$. Schedulabitity analysis of real-time traffic in worldfip networks: An integrated approach. IEEE Transactions on Industrial Electronics.

Almeida, L., J.A.Fonseca and P. Fonseca (1999). Flexible time triggered communication system based on the controler area network: Experimental results. Proc. Of 3th IFAC Int. Conf. On Fieldbus Systems and their Applications FeT'99 1, 24-31. 


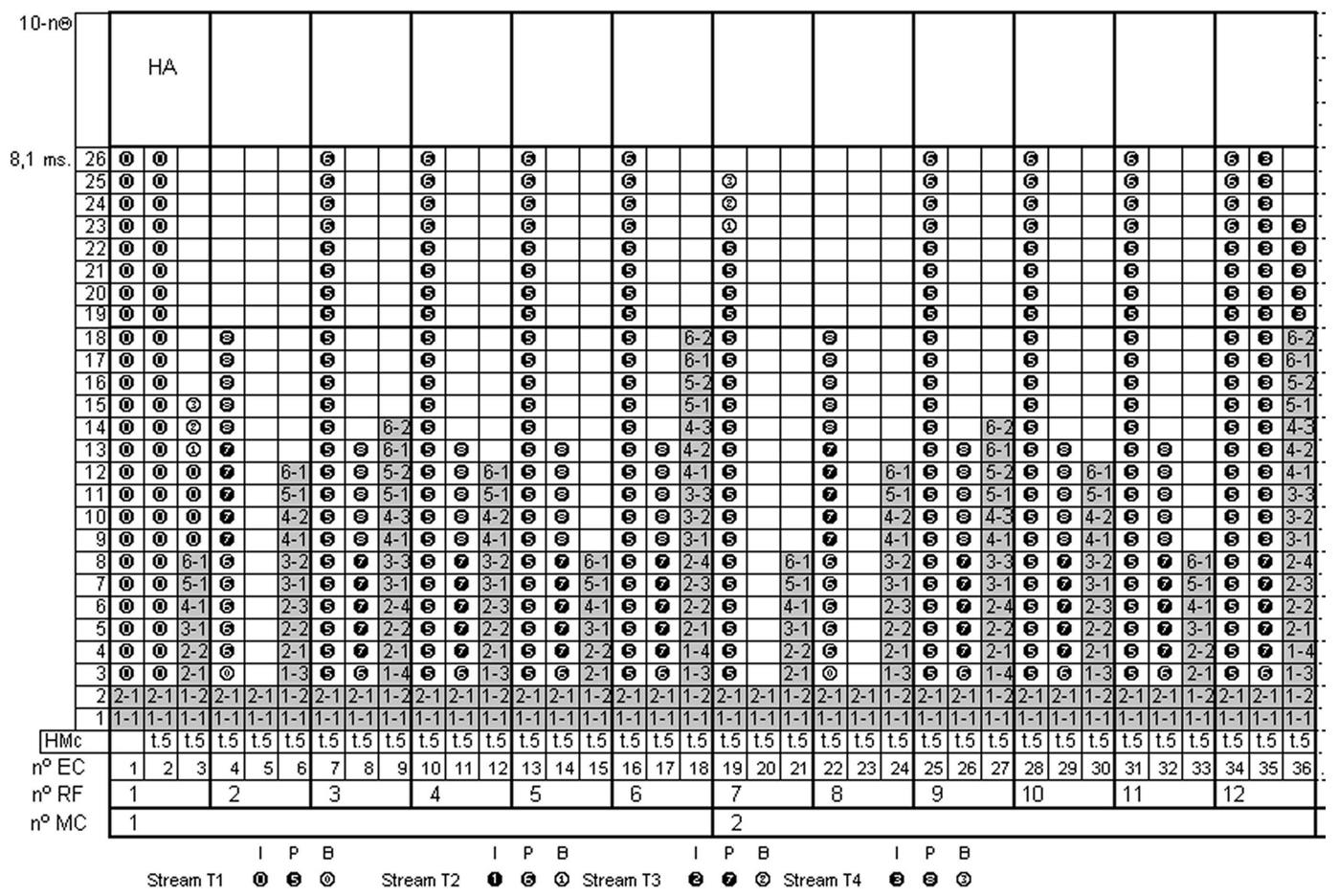

Fig. 3. Output of the scheduling algorithm with the input given in tables 2 and 3

Almeida, L., P. Pedreiras and J.A. Fonseca $(2002 b)$. The ftt-can protocol; why and how.. IEEE Transactions on Industrial Electronics 49(6), 1189-1201.

Chan, M., G. Pacifi and R. Stadler (1995). Managing real-time services in multimedia networks using dynamic visualization and high level controls. Proc. of ACM Multimedia, November 5-9, San Francisco, CA.

Feng, F., P. Zhao and A. Kumar (1996). Bounding application to application delays for multimedia traffic in fddi-based communications. Proc. of Multimedia Computing and Networking 1996, San Jose, Jan. pp. 174-185.

Han, C-C., K.G. Sing and C.J. Hou (2001). Synchronous bandwidth allocation for realtime communications with timed-token mac protocol. IEEE Transactions on Computers 50(5), 414-431.

Kee-Yin, J. (1999). Mpeg transmision schemes for a timed token medium access control network. ACM SIGCOMM Computer Communication Review 29(1), 66-80.

Lazar, A.A. and R. Stadlelr (1993). On reducing the complexity of management and control of broadband networks. Proc. of the Workshop on Distributed Systems: Operations and Management.

Pedreiras, P., L. Almeida and P. Gai (2002). The ftt-ethernet protocol: Merging flexibility, timeliness and efficiency.. Proc. Of 14th Euromicro Conference on Real-Time Systems (ECRTS'02).

Sempere, V. and J. Silvestre (2001). Image transport system in profibus networks. Proc. Of 4th IFAC Int. Conf. On Fieldbus Systems and their Applications FeT'01 pp. 24-31.

Sempere, V. and J. Silvestre (2003). Multimedia applications in industrial networks: Integration of image processing in profibus. IEEE Transactions on Industrial Electronics.

Silvestre, J., V. Sempere and M. Montava (2002). Optimization of the capacity of profibus for the transmission of images and control traffic. Proc. of 4 th IEEE Workshop on Factory Communication Systems WFCS'02 pp. 133140.

Stankovic, J et al. (1996). Strategic directions in real-time and embedded systems. $A C M$ Computing Surveys 28(4), 751-763.

Thomesse, J.P. (1998). The fieldbuses. Annual Reviews in Control 22, 35-45.

Tovar, E. and F. Vasques (1999). Cycle time properties of the profibus timed token protocol. Computer Communcation 11(13), 1206-1216.

Tovar, E., F. Vasques, F. Pacheco and L. Ferreira (2001). Industrial multimedia over factoryfloor networks. Proceedings of the 10th IFAC Symposium on Information Control Problems in Manufacturing (INCOM '01), Vienna, Austria pp. 20-22.

$\mathrm{Wu}$, C. and J.D. Irwin (1998). Multimedia and multimedia communication: A tutorial. IEEE Transactions on Industrial Electronics 45(1), 4-14.

Zheng, Q. and K.G. Shin (1993). Synchronous bandwidth allocation in fddi networks. Proc. of ACM Multimedia, 1993. 